\title{
Time and Frequency Resolved THz Spectroscopy of Micro- and Nano-Systems
}

\author{
J. Kröll, J. DARmo And K. Unterrainer \\ Institut für Photonik, Technische Universität Wien \\ Gusshausstrasse 27-29, 1040 Vienna, Austria
}

\begin{abstract}
Terahertz time-domain spectroscopy is applied to characterize ultrathin metallic layers and nanoscale composite material — single-wall carbon nanotube mat.
\end{abstract}

PACS numbers: 68.60.-p

\section{Introduction}

Terahertz technology [1], at present a dynamically developing field, was enabled by advances in ultra-short pulse lasers and semiconductor coherent sources in the frequency range of 1-50 THz. The extensive activities are focused on $\mathrm{THz}$ optics, spectrally bright sources, and detectors. The terahertz time-domain spectroscopy and terahertz imaging are main fields, where terahertz technology finds application. In this contribution we present our recent results in the field of terahertz time-domain spectroscopy (THz-TDS) applied to micro- and nano- systems and in the development of $\mathrm{THz}$ optics.

First, we report on a broadband absorptive anti-reflection coating that suppresses the reflection of terahertz pulses within an electro-optic detector. The anti-reflection coating is made of a thin chromium layer. We prove functionality of the layer in the $\mathrm{THz}$ time-domain spectroscopy. We estimate for the first time the complex index of refraction of such layers in the spectral range of 0.1-4 THz. Second, in the contribution we also report on a complex permittivity of nanoscale composite material - the single-wall carbon nanotube (SWCNT) mats measured by THz-TDS at frequencies from $0.1 \mathrm{THz}$ up to $4 \mathrm{THz}$.

\section{THz time-domain spectroscopic set-up}

The samples were evaluated by $\mathrm{THz}$ time-domain spectroscopy [2]. In our laboratory we have built such a system. It uses a Ti:sapphire laser with 80 femtosecond long near infrared (NIR) pulses to generate coherent $\mathrm{THz}$ radiation from 


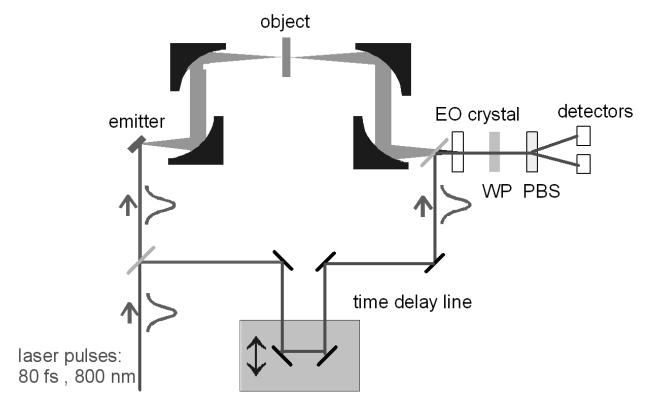

Fig. 1. Schematic of the terahertz time-domain spectroscopy set-up.

a biased GaAs emitter [3] and to gate an electro-optic GaP detector [4]. With this system a bandwidth of $7 \mathrm{THz}$ and signal-to-noise ratio of 5000 is reached for a single time-domain scan. The THz-TDS has advantages over conventional FTIR spectroscopy due to a higher sensitivity at lower frequencies $\left(<100 \mathrm{~cm}^{-1}\right)$, a spatial resolution (at the diffraction limit), and the unique possibility of timeresolved measurements of the absorption/reflection spectra [5]. The schematics of the set-up is in Fig. 1.

\section{Metallic anti-reflection coating}

In the THz-TDS the spectroscopic information is extracted from the time-domain signal by fast Fourier transformation. The validity of the obtained results depends strongly on the quality of the original time-domain signal. The refractive index mismatch at the interface electro-optic crystal/air leads to the appearance of multiple time delayed reflections of the main $\mathrm{THz}$ pulse in the signal trace if an electro-optic detector is used [6-8]. As known from the Fourier algorithm theory, any reflection within the signal trace manifests itself as modulation of the original spectrum. The amplitude of the modulation is proportional to the amplitude of the reflection and the modulation frequency depends on the time delay of the reflection. Such modulation disturbs significantly the information gained from the calculated spectrum.

At present the problem of $\mathrm{THz}$ pulse reflections in the electro-optic detector is solved by the use of thick electro-optic crystal substrates by postponing the reflection in time domain [9]. The reflections could be completely eliminated by the use of a dielectric quarter-wave thick $(\lambda / 4)$ layer. Such solution, however, would require a layer thickness of several tens of $\mu \mathrm{m}$ for $\lambda=100-300 \mu \mathrm{m}$ $(\sim 1-3 \mathrm{THz})$, and does not cover full frequency bandwidth of few-cycle $\mathrm{THz}$ pulses. Another possibility to eliminate the reflection at the crystal/air interface is to match their impedance by a properly chosen thin conductive layer: We model the interface of two different optical media as a junction of two transmission lines. To match their wave impedance, and hence to suppress the reflection at the junction, the theory of electrical circuits suggests to insert a shunting impedance 
between these transmission lines. Such shunting impedance $Z$ has to satisfy the relation $Z=\left|Z_{W_{2}} Z_{W_{1}} /\left(Z_{W_{2}}-Z_{W_{1}}\right)\right|$, where $Z_{W 1}$ and $Z_{W 2}$ are the wave impedance of the optical media. The shunting impedance can be realized by a metallic layer with a convenient resistivity and thickness. The sheet impedance $Z$ of such layer in the frame of the Drude model of metal's dielectric response [10]

$$
|Z|=\frac{1}{d \sigma_{\mathrm{DC}}} \sqrt{1+\frac{\omega^{2}}{\beta^{2}}},
$$

where $d$ is the metal layer thickness, $\sigma_{\mathrm{DC}}$ is the metal low frequency conductivity, and $1 / \beta$ is the scattering time of the electrons in the metal.

The material of choice for the anti-reflection layer can be any metal with medium conductivity $\sigma_{\mathrm{DC}}$. We have chosen chromium, which exhibits long-term stability of the parameters and compactness of the layer even at sub-10 nm thickness. With respect to the generally known dependence of the material parameters (e.g. resistivity) of thin layers on the film's thickness, we had to estimate the optimal layer's thickness experimentally. Therefore, we prepared a set of Cr layers of different thickness deposited on high-resistive silicon substrate. Figure 2a shows $\mathrm{THz}$ pulses reflected from the system silicon/Cr/air, which appears about $8.7 \mathrm{ps}$ after the main pulse due to a round trip of the $\mathrm{THz}$ pulse in the $0.4 \mathrm{~mm}$ thick silicon substrate. The amplitude of the pulses has been corrected to account for the transmission and reflection losses at the silicon/air interface. The amplitude of the reflected and transmitted pulses is decreasing for thicker Cr layer (Fig. 2b) because the sheet resistance of the layer is decreasing. For a layer of thickness of $90 \AA$, the amplitude of the reflected $\mathrm{THz}$ pulse already becomes negative in full agreement with the theory [10]. By fitting the experimental data to the theoretical model we get an optimal thickness of the $\mathrm{Cr}$ anti-reflection layer of $77 \AA$. At this thickness the $\mathrm{Cr}$ layer absorbs about $62 \%$ of the $\mathrm{THz}$ radiation. The effective index of refraction $n$ of the thin $\mathrm{Cr}$ layer in the frequency range of $0.1-4 \mathrm{THz}$ was estimated to be $n=37+\mathrm{i} 85$.
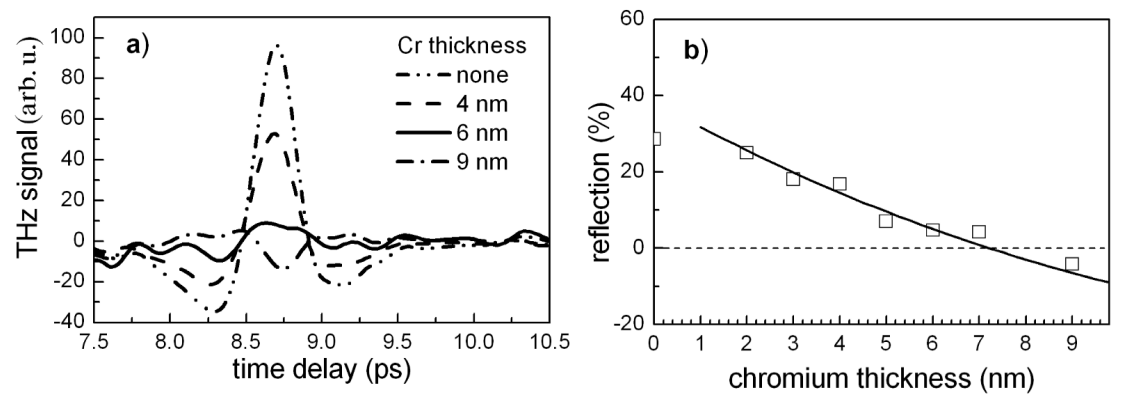

Fig. 2. Reflection of the $\mathrm{THz}$ pulses at the silicon/Cr/air interface system: (a) time window where the reflection appears (parameter — Cr layer thickness); (b) amplitude of the reflected $\mathrm{THz}$ pulse as a function of the Cr layer thickness. Solid line is a theoretical fit. 

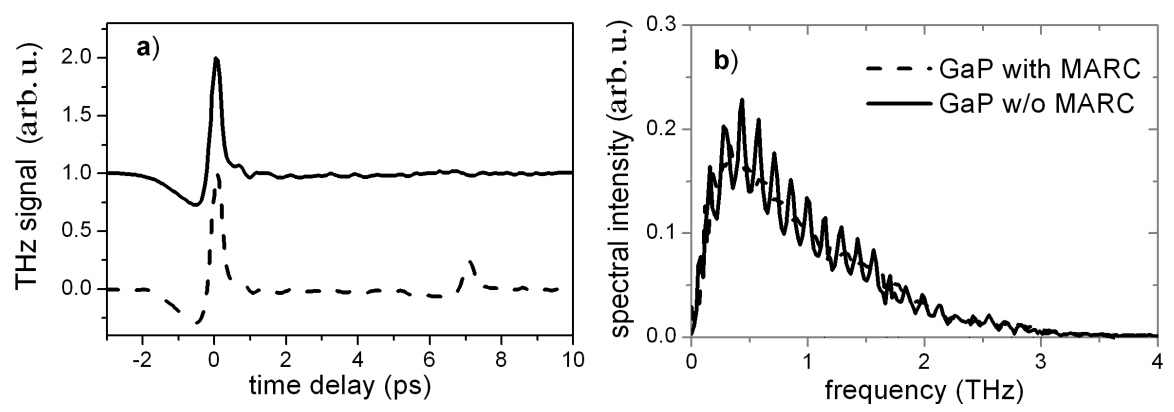

Fig. 3. Comparison of the $\mathrm{THz}$ signal measured by a GaP electro-optic detector with and without chromium anti-reflection coating: (a) time-domain data, (b) frequency-domain data.

We have applied the metallic anti-reflection coating (MARC) to a GaP electro-optic sensor. Figure 3a shows the time-domain $\mathrm{THz}$ signal generated from a photoconductive $\mathrm{THz}$ emitter [3] measured by a $300 \mu \mathrm{m}$ thick (110) oriented GaP crystal without and with the metallic anti-reflection layer. In the reference time-domain signal, a reflected $\mathrm{THz}$ pulse within the GaP sensor appears 7 ps after the main pulse (Fig. 3a). The amplitude of the reflected pulse is about $20 \%$ of the main $\mathrm{THz}$ pulse. The application of the metallic ARC reduces this reflection as can be seen in the time-domain signal. The residual reflection in the signal could still appear due to a systematic error in the control of the thickness of $\mathrm{Cr}$ films during the deposition on the GaP crystal and can be further improved. The comparison of the spectral intensity of the transmitted and the reference $\mathrm{THz}$ pulses shows a flat frequency dependence of the MARC properties in the whole frequency range of $0.1-4 \mathrm{THz}$ accessible in our set-up. The reduction of the reflection in the electro-optic crystal has a dramatic influence on the quality of the spectral data obtained by fast Fourier transformation of the time-domain signal as is demonstrated in Fig. 3b.

\section{Single-wall carbon nanotube films}

Carbon nanotubes and especially single-wall carbon nanotubes have attracted much attention in the last ten years due to their properties [11]. However, very little is known about the far infrared optical properties and phonon dynamics in these nanostructures. The Fourier transform infrared measurement was done by Uwaga et al. [12]. They observed excessive absorbency and the reflectance exhibits featureless Drude-like character in the frequency range of $0.5-150 \mathrm{THz}$. Hilt et al. [13] have studied charge transport in the SWCNT mats between 2.7 and $300 \mathrm{~K}$ for frequencies up to $0.5 \mathrm{THz}$. The plasma frequency, as well as the scattering rate, were found to be weak dependent on the temperature. The absorption has exhibited a transient region at about $200 \mathrm{GHz}$. Lately, Jeon and his coworkers $[14,15]$ have employed a novel progressive spectroscopic method for the far infrared 
region - terahertz time-domain spectroscopy (THz-TDS). Using THz-TDS they measured the absorption and the refractive index of the SWCNT mats at room temperature in the frequency range of 0.1-2.0 THz. They observed a monotonic increase in the absorption with frequency. All authors describe the SWCNT mats by an effective medium model with Drude and Lorentzian absorption [16]. However, there is a lack of full consistency of the model and the experimental data because of limited frequency range in which experiments were performed.
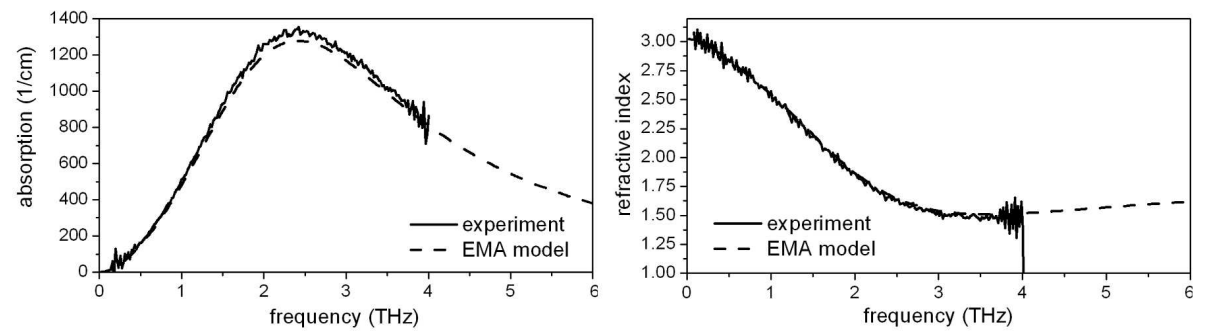

Fig. 4. Absorption and refractive index of SWCNT layer measured by THz-TDS.

The SWCNT mats were measured in the THz-TDS system built in our laboratory described earlier. Since for the SWCNT mats an excessive absorption at $\mathrm{THz}$ frequencies is typical [14, 15], we have dispersed nanotubes deposited onto highly resistive silicon substrate and employed a combination of transmission/reflection measurement. This allowed us to obtain a frequency resolved absorption also for highly absorptive SWCNTs samples (Fig. 4). In coherence with the theory prediction [16], power absorption roll-off was observed at frequencies above $2 \mathrm{THz}$ (see Fig. 3). This is the first consistent proof of the validity of effective media approximation (EMA) model for the SWCNT samples, because previous $\mathrm{THz}$ studies $[14,15]$ accessed only the monotonic increase in the $\mathrm{THz}$ absorption with frequency due to very limited bandwidth of their experimental set-up.

The frequency dependence of the real and imaginary part of the refractive index of SWCNT mat was fitted with the EMA model. The Table summarizes the parameters obtained for this model. We note quite a high volume filling factor of the SWCNT mat and large linewidth of the phonon response. Both parameters suggest a high fraction of bundled nanotubes in the SWCNT mat. Finally, Fig. 5 presents the contribution of carrier and phonon components to the absorption at frequencies $0.1-10 \mathrm{THz}$ as obtained from the model parameters.

We performed also the measurement of temperature dependence of the dielectric response of SWCNTs mats. Data obtained from the measurement at temperatures between 4.2 and $300 \mathrm{~K}$ show that the absorption by the free carriers dominates at all temperatures. Negligible temperature dependence of SWCNTs mats' absorption suggests that the absorption of $\mathrm{THz}$ radiation is controlled mainly by gapless metallic nanotubes and bundles of nanotubes. Future THz absorption 
TABLE

Parameters used in the EMA model [16] to fit the experimental data.

\begin{tabular}{l|c}
\hline \hline Parameter & Value \\
\hline SWCNT permittivity & 5.5 \\
Carrier plasma frequency & $5.1 \mathrm{THz}$ \\
Carrier scattering time & $1.45 \mathrm{THz}$ \\
Lorentzian oscillator strength & $6.7 \mathrm{THz}$ \\
Phonon frequency & $1.90 \mathrm{THz}$ \\
Linewidth & $4.56 \mathrm{THz}$ \\
Layer filling factor & 0.80
\end{tabular}

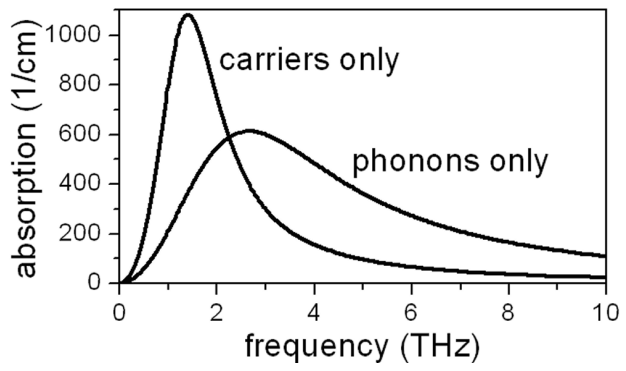

Fig. 5. Carrier and photon contribution to the absorption of a SWCNT layer obtained from the effective media approximation model fit to the experimental data.

experiments have to therefore include highly diluted SWCNTs samples with isolated nanotubes.

\section{Conclusions}

The capability of the terahertz time-domain spectroscopy was demonstrated on ultra-thin metallic layers and nano-composite material like the single-wall carbon nanotube mat. We have analyzed a $\mathrm{THz}$ response of ultra-thin chromium layer. The index of refraction of the thin $\mathrm{Cr}$ layer is effectively frequency independent in the $\mathrm{THz}$ frequency range of $0.1-4 \mathrm{THz}$. Such metallic layer can be successfully used as a broad-band absorptive anti-reflection coating and the functionality of $\mathrm{Cr}$ anti-reflection coating was demonstrated on an electro-optic $\mathrm{THz}$ detector.

The analysis of the dielectric response of single wall carbon nanotube mats in the frequency range of $0.1-4 \mathrm{THz}$ was also performed. The obtained data prove the validity of the model proposed earlier [16] according to which the free carriers and phonons contribute at those frequencies to the response. Because of non-negligible fraction carbon nanotubes with metallic character in the mats and due to the strong nanotubes bundling tendency, the effect of the free carriers dominates $\mathrm{THz}$ conductivity even at the low temperatures. The obtained results on 
SWCNTs mats are needed for future time-resolved $\mathrm{THz}$ absorption experiments. Such experiment will provide information about dynamics of carriers in individual SWCNTs (Drude part of absorption), as well as about phonon relaxation therein (Lorentzian part) and will answer the questions of consistency of the experimental observations on dynamics of photoexcited carriers and phonon modes using the high energy photons $[17,18]$.

\section{Acknowledgment}

The authors acknowledge financial support from the Austrian Fonds zur Förderung des Wissenschaftlichen Forschung (SFB-ADLIS). Furthermore, M. Hulman and Prof. H. Kuzmany (University of Vienna) are acknowledged for the SWCNTs samples.

\section{References}

[1] D. Mittleman, Sensing with Terahertz Radiation, Springer, Berlin 2003.

[2] D. Grischkowsky, S. Keiding, M. van Exter, Ch. Fattinger, J. Opt. Soc. Am. 7, 2006 (1990).

[3] J. Darmo, T. Müller, G. Strasser, K. Unterrainer, G. Tempea, Electron. Lett. 39, 460 (2003).

[4] J. Kröll, J. Darmo, K. Unterrainer, Electron. Lett. 40, 763 (2004).

[5] P.Y. Han, M. Tani, M. Usami, S. Kono, R. Kersting, X.-C. Zhang, J. Appl. Phys. 89, 2357 (2001).

[6] Q. Wu, X.-C. Zhang, Appl. Phys. Lett. 68, 1604 (1996).

[7] A. Nahata, A. Weling, V. Heinz, Appl. Phys. Lett. 69, 2321 (1996).

[8] Q. Wu, X.-C. Zhang, Appl. Phys. Lett. 70, 1784 (1997).

[9] A. Leitenstorfer, S. Hunsche, J. Shah, M.C. Nuss, W.H. Knox, Appl. Phys. Lett. 74, 1516 (1999).

[10] M. Born, E. Wolf, Principles of Optics, Cambridge Press, Cambridge 1999, ch. 14, p. 735 .

[11] R. Saito, G. Dresselhaus, M.S. Dresselhaus, Physical Properties of Carbon Nanotubes, Imperial College Press, London 1998.

[12] A. Uwaga, A.G. Rinzler, D.B. Tanner, Phys. Rev. B 60, R11305 (1999).

[13] O. Hilt, H.B. Brom, M. Ahlskog, Phys. Rev. B 61, R5129 (2000).

[14] T.-I. Jeon, K.-J. Kim, C. Kang, S.-J. Oh, J.-H. Son, K.H. An, D.J. Bae, Y.H. Lee, App. Phys. Lett. 80, 3403 (2002).

[15] T.-I. Jeon, K.-J. Kim, C. Kang, I.-H. Maeng, J.-H. Son, K.H. An, J.Y. Lee, Y.H. Lee, J. App. Phys. 94, 5736 (2004).

[16] J. Han, Z. Zhu, Z. Wang, W. Zhang, L. Yu, L. Sun, T. Wang, F. He, Y. Liao, Phys. Lett. A 310, 457 (2003).

[17] J.S. Lauret, C. Voisin, G. Cassabois, C. Delalande, Ph. Roussignol, O. Jost, L. Capes, Phys. Rev. Lett. 90, 057404 (2003).

[18] Y.Z. Ma, J. Stenger, J. Zimmermann, S.M. Bachilo, R.E. Smalley, R.B. Weisman, G.R. Fleming, J. Chem. Phys. 120, 3368 (2004). 